The effective index method and its application to semiconductor lasers

\title{
Buus, Jens
}

\section{Published in:}

I E E E Journal of Quantum Electronics

Link to article, DOI:

10.1109/JQE.1982.1071659

Publication date:

1982

Document Version

Publisher's PDF, also known as Version of record

Link back to DTU Orbit

Citation (APA):

Buus, J. (1982). The effective index method and its application to semiconductor lasers. I E E E Journal of Quantum Electronics, 18(7), 1083-1089. https://doi.org/10.1109/JQE.1982.1071659

\section{General rights}

Copyright and moral rights for the publications made accessible in the public portal are retained by the authors and/or other copyright owners and it is a condition of accessing publications that users recognise and abide by the legal requirements associated with these rights.

- Users may download and print one copy of any publication from the public portal for the purpose of private study or research.

- You may not further distribute the material or use it for any profit-making activity or commercial gain

- You may freely distribute the URL identifying the publication in the public portal

If you believe that this document breaches copyright please contact us providing details, and we will remove access to the work immediately and investigate your claim 
[12] H. Namizaki, "Transverse-junction-stripe lasers with a GaAs p-n homojunction," IEEE J. Quantum Electron., vol. QE-11, pp. 427-431, July 1975.

[13] K. Aiki, M. Nakamura, T. Kuroda, R. Ito, N. Chinone, and M. Maeda, "Transverse mode stabilized $\mathrm{Al}_{x} \mathrm{Ga}_{1-x}$ As injection lasers with channelled-substrate-planar structure," $J$. Quantum Electron., vol. QE-14, pp. 89-94, Feb. 1978.

[14] K. Saito and R. Ito, "Buried-heterostructure AlGaAs lasers," IEEE J. Quantum Electron., vol. QE-16, pp. 205-215, Feb. 1980.

[15] H. G. Danielmeyer, "Effects of drift and diffusion of excited states on spatial hole burning and laser oscillation," J. Appl. Phys., vol. 42, pp. 3125-3132, July 1971, and W. Streifer, R. D. Burnham, and D. R. Scifres, "Dependence of longitudinal mode structure on injected carrier diffusion in diode lasers," IEEE $J$. Quantum Electron., vol. QE-13, pp. 403-404, June 1977.

[16] L. W. Casperson, "Threshold characteristics of multimode laser oscillators," J. Appl. Phys., vol. 46, pp. 5194-5201, Dec. 1975.

[17] J. A. Copeland, "Single-mode stabilization by traps in semiconductor lasers," IEEE J. Quantum Electron., vol. QE-16, pp. 721-727, July 1980.

[18] D. Kasemset, S. Rotter, and C. G. Fonstad, "Liquid phase epitaxy of PbTeSe lattice-matched to PbSnTe," J. Electron. Mat., vol. 10, pp. 863-878, Sept. 1981.

Dumrong Kasemset was born in Bangkok, Thailand, on March 2, 1954. He received the S.B. degree in 1977, the S.M. and E.E. degrees in 1979, and the Ph.D. degree in 1981, all from the Massachusetts Institute of Technology, Cambridge.

He has authored and coauthored a number of publications in the field of lead-salt semiconductor lasers. He is presently working at the Rockwell Science Center, Thousand Oaks, CA.

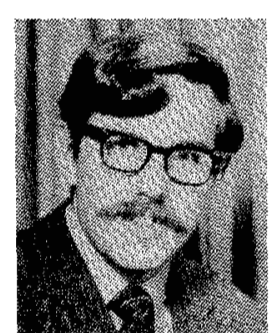

Clifton G. Fonstad, Jr. (S'63-M'66-M'74) received the B.S. degree from the University of Wisconsin, Madison, in 1965, and the M.S. and $\mathrm{Ph} . \mathrm{D}$. degrees in electrical engineering from the Massachusetts Institute of Technology, Cambridge, in 1966 and 1970 , respectively.

He joined the M.I.T. faculty in September 1970 and is now a full Professor. He directs a research group with which he is studying the growth and characterization of III-V ternary and quaternary compounds, including InGaAs and InGaAsP, and the fabrication of heterostructure devices such as bipolar transistors and guided wave optical circuitry from these materials. The group is also concerned with the lattice-matched $\mathrm{Pb}$ salt heterostructure system $\mathrm{PbSnTe} / \mathrm{PbTeSe}$ and with the fabrication and modeling of buried heterostructure lasers, and other single-mode diode laser geometries, in the $\mathrm{Pb}$ salts.

Dr. Fonstad is a member of the American Physical Society and the Electrochemical Society.

\title{
The Effective Index Method and Its Application to Semiconductor Lasers
}

\author{
JENS BUUS, MEMBER, IEEE
}

\begin{abstract}
By the effective index method a two-dimensional field problem is transformed to a problem for a one-dimensional effective waveguide. This method is applied to semiconductor lasers having a gradual lateral variation in the complex permittivity. For the special case of a parabolic variation, analytical formulas for the required gain in the center and the half width of the intensity distribution are derived. The results are compared with a numerical method and very good agreement is found except in some cases where convergence problems occur for the numerical method. This agreement is taken as evidence for the validity of results obtained using the effective index method for analysis of semiconductor laser structures.
\end{abstract}

\section{INTRODUCTION}

$\mathrm{T}$ HE effective index method was originally proposed for analysis of rectangular dielectric waveguides [1]. By this method a two-dimensional problem is transformed to a problem for a one-dimensional effective waveguide; this latter problem can then be solved analytically or by simpler methods

Manuscript received December 14, 1981; revised March 15, 1982.

The author is with the Electromagnetics Institute, Technical University of Denmark, Lyngby, Denmark. than required for the two-dimensional case. The method has been shown to be better than other simple methods for rectangular dielectric waveguides.

The analysis of waveguiding in semiconductor lasers is a twodimensional problem and the effective index method has been used in different forms to simplify the solution of this problem, e.g., [2] -[6] .

In this paper some general formulas related to the analysis of waveguiding in laser structures are presented. For the special case of parabolic permittivity variation the general formulas are used to derive analytical expressions for the gain in the center of the active region required to overcome the loss, and for the full width at half intensity of the field distribution in the junction plane.

For the parabolic profile a numerical method was presented in [7], this method is summarized in Section III and it is shown how the method can be somewhat simplified, reducing the required computing time considerably.

Finally, in Section IV the results from the two methods are presented and discussed. 


\section{Field Calculation By the \\ "EFFECtive Index Method"}

\section{A. Slab Structure}

We first consider a simple slab structure with permittivity variation in the $x$-direction only, as shown in Fig. 1. Using the scalar wave equation we find the propagation constant for the TE modes ( $y$ polarization) (e.g., $[8])$

$$
\left(\frac{\beta_{z}}{k}\right)^{2}=\epsilon_{\mathrm{eff}}=b \epsilon_{1}+(1-b) \epsilon_{2}
$$

where the normalized propagation constant $b$ is the solution to the characteristic equation, which for the fundamental mode reads

$$
\begin{aligned}
v \sqrt{1-b} & =\arctan \sqrt{\frac{b}{1-b}} \\
v & =k t \sqrt{\epsilon_{1}-\epsilon_{2}} .
\end{aligned}
$$

The imaginary parts of $\epsilon_{1}$ and $\epsilon_{2}$ are much smaller than the real parts and hence we consider the imaginary parts as perturbations of the corresponding slab structure with real indexes. This gives for the effective refractive index

$$
n_{\mathrm{eff}}=\operatorname{Re}\left\{\sqrt{\epsilon_{\mathrm{eff}}}\right\}=\frac{1}{k} \operatorname{Re}\left\{\beta_{z}\right\}=\sqrt{b_{o} n_{1}^{2}+\left(1-b_{o}\right) n_{2}^{2}}
$$

where $b_{o}$ is the solution to the characteristic equation (2) with the normalized frequency $v$ replaced by the real number $v_{o}$

$$
v_{o}=k t \sqrt{n_{1}^{2}-n_{2}^{2}} \text {. }
$$

We now include the imaginary parts of $\epsilon_{1}$ and $\epsilon_{2}$ and get a complex value for $b$ with $b_{i}=\operatorname{Im}\{b\} \ll b_{o}$, i.e.,

$$
b=b_{o}+j b_{i}=b_{o}+\left.\Delta v \frac{d b}{d v}\right|_{v=v_{o}}
$$

where $\Delta v$ is the change in $v$ due to the imaginary parts of $\epsilon_{1}$ and $\epsilon_{2}$

$$
\Delta v=j \frac{v_{0}}{n_{1}^{2}-n_{2}^{2}} \frac{n_{1} g+n_{2} \alpha}{2 k} .
$$

This gives a complex value for $\epsilon_{\text {eff }}$ when (6) is used in (1), and we introduce an effective gain $g_{\text {eff }}$ defined by (neglecting $\left.\left(g_{\text {eff }} / k\right)^{2}\right)$

$$
\epsilon_{\mathrm{eff}}=n_{\mathrm{eff}}^{2}+j \frac{n_{\text {eff }} g_{\mathrm{eff}}}{k} .
$$

Use of (6) and (7) in (1) gives

$$
g_{\text {eff }}=2 \operatorname{Im}\left\{\beta_{z}\right\}=k \frac{\operatorname{Im}\left\{\epsilon_{\text {eff }}\right\}}{n_{\text {eff }}}=\frac{\Gamma n_{1} g-(1-\Gamma) n_{2} \alpha}{n_{\text {eff }}}
$$

where the weighing factor $\Gamma$ is given by

$$
\Gamma=b_{o}+\left.\frac{v_{o}}{2} \frac{d b}{d v}\right|_{v=v_{o}}=b_{o}+\left(1-b_{o}\right) \frac{v_{o} \sqrt{b_{o}}}{1+v_{o} \sqrt{b_{o}}} .
$$

$\Gamma$ is also defined as the fraction of the intensity propagating

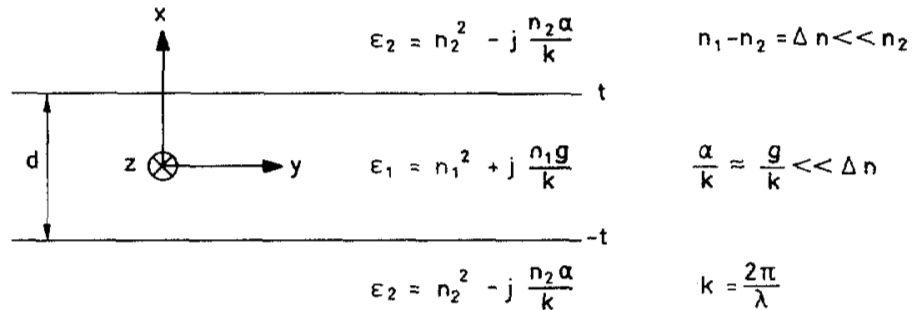

Fig. 1. Definition of slab structure with $\epsilon$ denoting permittivity, $n$ refractive indexes, $\alpha$ loss, $g$ gain, and $k$ wavenumber. The terms $(\alpha / k)^{2}$ and $(g / k)^{2}$ are neglected.

in the guiding layer; direct calculation gives the same expression as (10).

Equation (9) can alternatively be derived in the following way [9] : the scalar field equation is multiplied by the complex conjugate of the field; the resulting expression is integrated from $-\infty$ to $\infty$. This gives a variational expression for $\beta_{z}^{2}$, and taking the imaginary parts of $\epsilon_{1}$ and $\epsilon_{2}$ as perturbations, the field (to first order) remains real whereas $\beta_{z}$ becomes complex, taking the imaginary part of the expression for $\beta_{z}^{2}$ gives: $E(x)$ being the field distribution

$$
\operatorname{Im}\left\{\epsilon_{\mathrm{eff}}\right\}=\operatorname{Im}\left\{\frac{\beta_{z}^{2}}{k^{2}}\right\}=\frac{\int_{-\infty}^{\infty} \operatorname{Im}\{\epsilon(x)\}|E(x)|^{2} d x}{\int_{-\infty}^{\infty}|E(x)|^{2} d x}
$$

defining $\Gamma$ as the fraction of the intensity between $-t$ and $t$ (9) is obtained. Since $n_{2} \leqslant n_{\text {eff }} \leqslant n_{1}$ and $\left(n_{1}-n_{2}\right) \ll n_{2} \lesssim$ $n_{1}$ (9) gives

$$
g_{\text {eff }} \approx \Gamma g-(1-\Gamma) \alpha .
$$

\section{B. Slab with Lateral $\epsilon$ Variation}

We now consider the case where $\varepsilon_{1}$ and $\epsilon_{2}$ are functions of $y$ given by

$$
\left.\begin{array}{l}
\epsilon_{1}(y)=\epsilon_{1}(0)-f_{1}(y) \\
\epsilon_{1}(0)=n_{1}^{2}+j \frac{n_{1} g_{o}}{k} \\
f_{1}(0)=0 \\
\epsilon_{2}(y)=\epsilon_{2}(0)-f_{2}(y) \\
\epsilon_{2}(0)=n_{2}^{2}-j \frac{n_{2} \alpha}{k} \\
f_{2}(0)=0 .
\end{array}\right\}
$$

We define an effective permittivity for each plane given by a value of $y$, according to (1)

$$
\epsilon_{\mathrm{eff}}(y)=b(y) \epsilon_{1}(y)+(1-b(y)) \epsilon_{2}(y) .
$$

For the laser structures considered we have

$$
\left|f_{1}(y)\right|,\left|f_{2}(y)\right| \ll\left|\epsilon_{1}(0)-\epsilon_{2}(0)\right| ;
$$

hence, we only use one term in the expressions of $b$

$$
b(y)=b(0)+\left.(v(y)-v(0)) \frac{d b}{d v}\right|_{y=0} .
$$


Insertion of (16) and (13) in (14) then gives for the variation of $\epsilon_{\mathrm{eff}}$ in the $y$ direction

$$
\begin{aligned}
\Delta(y)= & \epsilon_{\mathrm{eff}}(0)-\epsilon_{\mathrm{eff}}(y) \\
= & b(0) f_{1}(y)+\left.(v(0)-v(y)) \frac{d b}{d v}\right|_{y=0} \epsilon_{1}(0) \\
& +(1-b(0)) f_{2}(y)-\left.(v(0)-v(y)) \frac{d b}{d v}\right|_{y=0} \epsilon_{2}(0) \\
v(y)= & k t \sqrt{\left(\epsilon_{1}(0)-f_{1}(y)\right)-\left(\epsilon_{2}(0)-f_{2}(y)\right)} \\
\approx & v(0)\left(1-\frac{1}{2} \frac{f_{1}(y)-f_{2}(y)}{\epsilon_{1}(0)-\epsilon_{2}(0)}\right) .
\end{aligned}
$$

Using the expression for the derivative of $b$ with respect to $v$ [also used in (10)] (17) and (18) give

$$
\Delta(y)=\Gamma f_{1}(y)+(1-\Gamma) f_{2}(y) .
$$

In (19) $\Gamma$ is the filling factor for the simple real slab guide, i.e., (10) with $v_{o}$ given by (5) and hence independent of $y$. This formula, of course, is valid only when the expansion (16) is valid, i.e., (15) must be satisfied. If this is not the case the definition of $\epsilon_{\mathrm{eff}}(y)$ given in (14) must be used directly.

The result (19) is also derived in [10], [11] and was first used in [3]. In [12] the $y$ dependence and imaginary part of $b$ is neglected, thus giving an equation similar to (19) but with $\Gamma$ replaced by $b_{o}$. When the imaginary part of $b$ is included (but the $y$ dependence ignored) one again gets an equation similar to (19), but with $b_{o}$ as weighing factor for the real part and $\Gamma$ for the imaginary part [5], [13]. If the guiding parallel to the active layer is dominated by variations in the thickness of the active layer we get [2]

$$
\Delta(y)=(b(0)-b(y))\left(\epsilon_{1}(0)-\epsilon_{2}(0)\right) .
$$

In this case, the imaginary part of $b$ and the $y$ dependence of $\epsilon_{1}$ and $\epsilon_{2}$ are unimportant for the guiding.

The effective index method was originally proposed for rectangular structures [1]. The method is shown [14] to be better than another simple method [15] for rectangular dielectric waveguides.

The $y$ variation of the effective index gives a $y$ variation of the field which can be described by a lateral propagation constant $\beta_{y}\left(\left|\beta_{y}\right| \ll\left|\beta_{z}\right|\right.$ if (15) is satisfied). Compared with the slab without variations in the $y$ direction we now get modified values of the propagation constant, the effective index, and the effective gain (mode gain), which we define as

$$
\begin{aligned}
\beta_{z}^{\prime} & =\sqrt{\beta_{z}^{2}-\beta_{y}^{2}} \\
n_{\mathrm{eff}}^{\prime} & =\frac{1}{k} \operatorname{Re}\left\{\beta_{z}^{\prime}\right\} \\
G & =2 \operatorname{Im}\left\{\beta_{z}^{\prime}\right\}
\end{aligned}
$$

where $\beta_{z}$ is the result for the slab guide without $y$ variations $\left(f_{1}(y)=f_{2}(y)=0\right)$. Expansion of the square root of $\beta_{z}^{\prime}$ in (22) and (23) and use of (4) and (9) gives

$$
\begin{aligned}
n_{\mathrm{eff}}^{\prime} & =n_{\mathrm{eff}}-\frac{\operatorname{Re}\left\{\beta_{y}^{2}\right\}}{2 n_{\mathrm{eff}} k^{2}} \\
G & =g_{\mathrm{eff}}-\frac{\operatorname{Im}\left\{\beta_{y}^{2}\right\}}{n_{\mathrm{eff}} k} .
\end{aligned}
$$

\section{The Parabolic Profile}

We now apply the results to the case of a parabolic variation of index and gain in the active layer [7]

$$
\left.\begin{array}{l}
f_{1}(y)=a^{2} y^{2} \\
f_{2}(y)=0
\end{array}\right\}
$$

hence from (19)

$$
\Delta(y)=\Gamma a^{2} y^{2}=a_{\mathrm{eff}}^{2} y^{2} .
$$

In order to introduce convenient parameters we assume that the gain in the active layer equals 0 at $y= \pm y_{o}$ (half gain width), i.e.,

$$
\epsilon_{1}\left(y_{o}\right)=n_{1}^{2}-2 n_{1} \delta n
$$

where $\delta n \ll\left(n_{1}-n_{2}\right)$ is the change of the real part of the refractive index going from $y=0$ to $y= \pm y_{o}$. Thus

$$
a^{2} y_{0}^{2}=\frac{n_{1} g_{o}}{k}(R+j)
$$

with the ratio $R$ between the variation in the real and imaginary parts of $\epsilon_{1}$ (note that $R$ is defined differently in [7])

$$
R=\delta n \frac{2 k}{g_{o}} \text {. }
$$

The effective guiding is described by $a_{\text {eff }}$

$$
a_{\mathrm{eff}}=\sqrt{\frac{\Gamma n_{1} g_{o}}{k y_{0}^{2}}(R+j)} .
$$

As is well known, the solution to the field equation with an effective permittivity variation given by (27) is HermiteGauss functions and the field variation in the $y$-direction for the fundamental mode becomes

$$
E(y)=e^{-k a_{\text {eff }}\left(y^{2} / 2\right)}
$$

with the intensity variation

$$
I(y)=|E(y)|^{2}=e^{-k \operatorname{Re}\left\{a_{\mathrm{eff}}\right\} y^{2}}
$$

and eigenvalue (square of lateral propagation constant)

$$
\beta_{y}^{2}=k a_{\text {eff }} \text {. }
$$

Thus (24) and (25) give

$$
\begin{gathered}
n_{\text {eff }}^{\prime}=n_{\text {eff }}-\frac{\operatorname{Re}\left\{a_{\text {eff }}\right\}}{2 n_{\text {eff }} k} \\
G=g_{\text {eff }}-\frac{\operatorname{Im}\left\{a_{\text {eff }}\right\}}{n_{\text {eff }}} .
\end{gathered}
$$

In the lasing state the required mode gain $G_{\text {req }}$ must balance mirror and internal scattering losses (i.e., losses not included in the net material gain); using (9), (36), and (31) this condition can be written as 


$$
\begin{aligned}
G_{\text {req }}= & \alpha_{\text {mirror }}+\alpha_{\text {scattering }}=\frac{\Gamma n_{1} g_{0}-(1-\Gamma) n_{2} \alpha}{n_{\text {eff }}} \\
& -\frac{\sqrt{\frac{\Gamma n_{1} g_{o}}{k y_{o}^{2}}} \operatorname{Im}\{\sqrt{R+j}\}}{n_{\text {eff }}}
\end{aligned}
$$

which can be rearranged as

$$
g_{o}-\sqrt{\frac{g_{o}}{\Gamma n_{1} k y_{o}^{2}}} \operatorname{Im}\{\sqrt{R+j}\}=G_{\infty}
$$

with $G_{\infty}$ being the required gain in the active region for $y_{0} \rightarrow \infty$

$$
\begin{aligned}
G_{\infty} & =\frac{G_{\text {req }}+(1-\Gamma) \alpha \frac{n_{2}}{n_{\text {eff }}}}{\Gamma \frac{n_{1}}{n_{\text {eff }}}} \\
& \approx \frac{G_{\text {req }}+(1-\Gamma) \alpha}{\Gamma} .
\end{aligned}
$$

We define a new parameter $C$ as

$$
C=\operatorname{Re}\{\sqrt{R+j}\}=\sqrt{\frac{R+\sqrt{1+R^{2}}}{2}}=\frac{1}{2 \operatorname{Im}\{\sqrt{R+j}\}} .
$$

It should be noted that $C$ is related to the astigmatism factor $K$, defined in [15]

$$
K=\sqrt{1+\frac{1}{4 C^{4}}} .
$$

The product of the width of the near and far fields can be shown to be proportional to $K$, for $R \gg 1$ (index guiding) $K=1$.

Solving (38) for $g_{o}$ and the use of (40) gives

$$
\begin{aligned}
g_{o} & =\frac{\left(1+\sqrt{\left.1+G_{\infty} \Gamma B C^{2}\right)^{2}}\right.}{\Gamma B C^{2}} \\
B & =16 n_{1} k y_{o}^{2} .
\end{aligned}
$$

Note that this formula is somewhat different from the corresponding formula (35) in [7] .

The full width of half intensity $w_{o}$ is found from

$$
I\left(\frac{w_{0}}{2}\right)=\frac{1}{2}
$$

which gives

$$
\begin{aligned}
w_{o} & =\frac{4 \sqrt{\ln 2} y_{o}}{\sqrt[4]{g_{o} \Gamma B C^{2}}} \\
& =\frac{4 \sqrt{\ln 2} y_{o}}{\sqrt{1+\sqrt{1+G_{\infty} \Gamma B C^{2}}}} .
\end{aligned}
$$

The expression (35) can now be written as

$$
n_{\mathrm{eff}}^{\prime}=n_{\mathrm{eff}}-\frac{2 \ln 2}{n_{\mathrm{eff}}\left(k w_{o}\right)^{2}} \text {. }
$$

Evaluation of (42) in the two limiting cases for small and large values of the thickness $d=2 t$ gives

$$
\begin{aligned}
& d \rightarrow 0 \Rightarrow w_{o} \rightarrow \frac{4 \sqrt{\ln 2} y_{o}}{\sqrt{1+\sqrt{1+\frac{n_{2}}{n_{1}}\left(G_{\text {req }}+\alpha\right) B C^{2}}}} \\
& d \rightarrow \infty \Rightarrow w_{1} \rightarrow \frac{4 \sqrt{\ln 2} y_{o}}{\sqrt{1+\sqrt{1+G_{\text {req }} B C^{2}}}} \\
& \frac{w_{o}(d \rightarrow \infty)}{w_{o}(d \rightarrow 0)} \leqslant \sqrt[4]{\frac{n_{2}{ }_{\frac{n_{1}}{}\left(G_{\text {req }}+\alpha\right)}^{G_{\text {req }}}}{}} .
\end{aligned}
$$

This result shows that $w_{o}$ is nearly independent of $d$. For small $y_{o}(46)$ gives

$$
w_{o} \approx \sqrt{2 \ln 2}\left(2 y_{o}\right) \text {. }
$$

\section{iII. Field Calculation by Planewave Expansion}

Following [7], the field in the active layer of a laser structure with the permittivity given by (26) and (29) is written (retaining a finite number of expansion terms)

$$
E(x, y)=\sum_{l=0}^{l_{\max }} A_{l} \cos \left(q_{l} x\right) H_{l}(\sqrt{a k} y) \exp \left(-a k \frac{y^{2}}{2}\right) l_{\text {even }} .
$$

$H_{l}$ is the Hermite polynomial of order $l$. Insertion of (52) in the field equation gives relations between $q_{l}$ and the propagation constant $\beta_{z}^{\prime}$

$$
k^{2} \epsilon_{1}(0)-\beta_{z}^{\prime 2}=q_{l}^{2}+a k(2 l+1) \quad l=0,2, \cdots, l_{\max } .
$$

Matching (52) to a planewave expansion at $x= \pm t$ (see Fig. 1) results in a set of linear equations for the expansion coefficients $A_{l}$ appearing in (52)

$$
A_{l}^{\prime}=\sum_{l} A_{l} \Omega_{l, l^{\prime}} \quad l=0,2, \cdots, l_{\max } .
$$

The matrix elements are calculated in [7] and can be written as

$$
\begin{aligned}
\Omega_{l, l^{\prime}} & =\frac{(-1)^{\left(l^{\prime}+l^{\prime}\right) / 2} \cos \left(q_{l} \frac{d}{2}\right)}{q_{l^{\prime}} \sin \left(q_{t^{\prime}} \frac{d}{2}\right)} \sqrt{\beta_{z}^{\prime 2}-\epsilon_{2} k^{2}} \cdot I_{l^{\prime}, l} \\
I_{l^{\prime}, l} & =\frac{1}{\sqrt{\pi} 2^{l^{\prime} l^{\prime} !}} \int_{-\infty}^{\infty} H_{l^{\prime}}(z) H_{l}(z) e^{-z^{2}} \sqrt{1+A z^{2}} d z \\
A & =\frac{a k}{\beta_{z}^{\prime 2}-\epsilon_{2} k^{2}} .
\end{aligned}
$$

In order to have solutions for (54) we must have

$$
\operatorname{Det}(\overline{\bar{\Omega}}-\overline{\bar{E}})=0 \text {. }
$$

This condition determines the propagation constant $\beta_{z}^{\prime}$ and the required center gain $g_{o}$ is determined by varying $g_{o}$ [and hence the value of $a$ given by (29)] until the condition $2 \operatorname{Im}\left\{\beta_{z}^{\prime}\right\}=G_{\text {req }}$ is satisfied, with $G_{\text {req }}$ being the mode gain required to overcome the loss.

Since a double iteration is needed in order to calculate $\beta_{z}^{\prime}$ and $g_{o}$, the computing time becomes excessive if $(55 b)$ is evaluated by numerical integration. The Hermite-Gauss part 
of the integrand in (55b) obtains its maximum value for $z=z_{\max } \approx \sqrt{l_{\max }}$ and decreases very rapidly for $z>z_{\max }$. If we have $|A| z^{\prime 2}<1$ with $z^{\prime} \approx \sqrt{l_{\max }}+1$ a power series expansion of the square root in $(55 b)$ will be valid in the interval of $z$ values where the integrand is nonvanishing, for $l_{\max }=10$ the condition is satisfied for all parameter values considered, this will not be the case for extremely small values of the thickness. Thus, retaining $m$ terms

$$
\sqrt{1+A z^{2}}=1+\sum_{i=1}^{m} b_{i} A^{i} z^{2 i}
$$

Expansion of the powers in Hermite polynomials ( $c_{i, n}$ given in [17])

$$
z^{2 i}=\sum_{n=0}^{i} c_{i, n} H_{2 n}(z)
$$

gives

$$
\sqrt{1+A z^{2}}=1+\sum_{i=1}^{m}\left\{b_{i} A^{i} \sum_{n=0}^{i}\left(c_{i, n} H_{2 n}(z)\right)\right\} .
$$

Equation (55b) now gives

$$
I_{l^{\prime}, l}=\delta_{l^{\prime}, l}+\frac{1}{\sqrt{\pi} 2^{l^{\prime}} l^{\prime} !} \sum_{i=1}^{m}\left\{b_{i} A^{i}\left(\sum_{n=0}^{i} c_{i, n} I_{2 n, l^{\prime}, l}\right)\right\}
$$

where $[18]$

$$
\begin{aligned}
I_{2 n, l^{\prime}, l} & =\int_{-\infty}^{\infty} \dot{H}_{2 n}(z) H_{l^{\prime}}(z) H_{l}(z) e^{-z^{2}} d z \\
& =\frac{\sqrt{\pi} 2^{s}(2 n) ! l^{\prime} ! l !}{(s-2 n) !\left(s-l^{\prime}\right) !(s-l) !}, \quad s=\frac{2 n+l^{\prime}+l}{2} .
\end{aligned}
$$

$I_{l^{\prime}, l}$ differ from 0 only if $\left|l^{\prime}-l\right| \leqslant 2 n \leqslant\left(l^{\prime}+l\right)$. Insertion of $(61)$ in (60) gives

$$
I_{l^{\prime}, l}=\delta_{l^{\prime}, l}+\sum_{i=1}^{m} A^{i} d_{i, l^{\prime}, l}
$$

The coefficients $d_{i, l^{\prime}, l}$ need only be calculated once

$$
d_{i, l^{\prime}, l}=\frac{l !}{2^{l^{\prime}}} b_{i} \sum_{n=0}^{i} c_{i, n} \frac{2^{s}(2 n) !}{(s-2 n) !\left(s-l^{\prime}\right) !(s-l) !} .
$$

This very fast method for calculation of the matrix elements has been compared with results obtained by numerical integration, and fine agreement was found. The computing time is now determined by the time required to solve (56) rather than $55 b$ ).

\section{RESULTS}

The center gain $g_{o}$ and full width at half intensity $w_{o}$ are first calculated by the effective index method according to (42) and (46). Some results are shown in Figs. 2 and 3.

The planewave expansion method is applied to the following cases: $n_{2}=3.4,3.5 ; R=-3,-2,-1,0,1 ; d=0.1,0.15,0.2$, $0.4 \mu \mathrm{m} ; y_{o}=2,4,6,8,10 \mu \mathrm{m}$, using $m=4,5$ in (57) and $l_{\max } \leqslant 10$ in $(52)$.

In contrast to [7], very good agreement with the effective

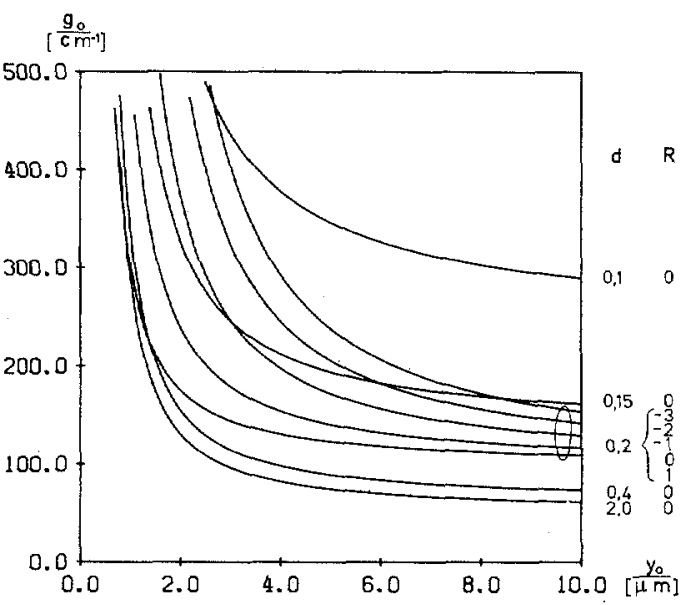

Fig. 2. Center gain $g_{o}$ as function of the gain width $y_{o}$ for various values of the active layer thickness $d$ and the guiding parameter $R$. Other parameter values: $\lambda=0.9 \mu \mathrm{m}, G_{\text {req }}=50 \mathrm{~cm}^{-1}, \alpha=20 \mathrm{~cm}^{-1}$, $n_{1}=3.6$, and $n_{2}=3.4$.

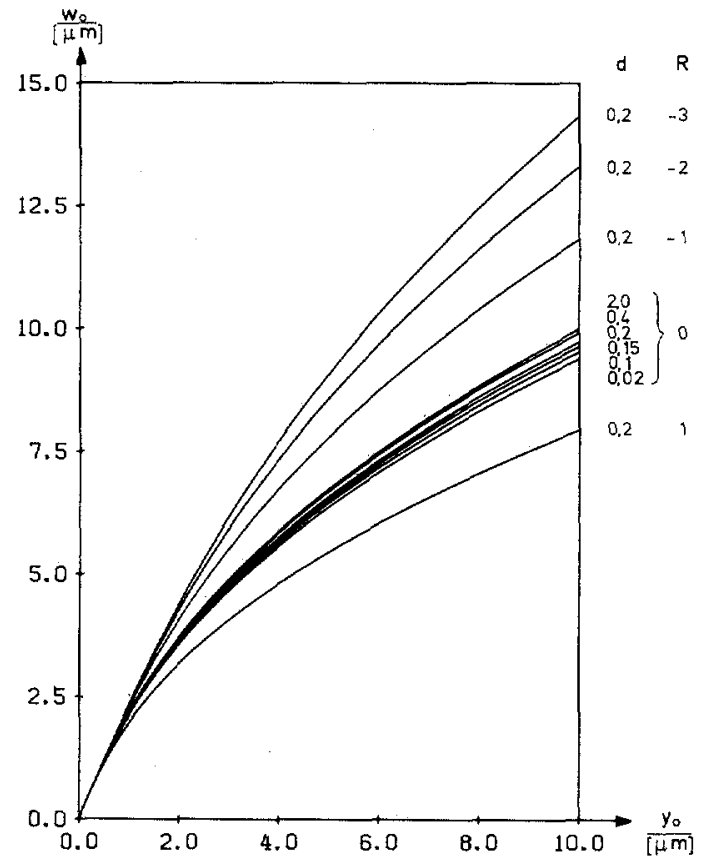

Fig. 3. Full width at half intensity $w_{o}$ calculated under the same conditions as $g_{0}$ in Fig. 1.

index method is found; a few examples are given in Table I.

Use of the simple (39b) instead of (39a) in the effective index calculation changes the values for $g_{o}$ in Table I by 4$5 \mathrm{~cm}^{-1}$ and $w_{o}$ by $0.05-0.06 \mu \mathrm{m}$, thus degrading the agreement with the planewave method significantly.

The normalized intensity distributions corresponding to three of the cases considered in Table I are shown in Fig. 4.

For $R=-1$ the result converges rapidly with an increasing number of expansion functions $\left[l_{\max }\right.$ in (52)], in the case of $R=-2$ a shoulder develops for $l_{\max } \geqslant 4$ and for $R=-3$ a large peak appears for $l_{\max } \geqslant 6$. This behavior is attributed to the complex parameter $a$ in (52), for increasing negative values of $R$ the real part of $a$ decreases and the imaginary part increases. It is to be noted, however, that a correct value of the width of half intensity is found also for large negative $R$, but a larger value for $l_{\max }$ is required to give a reliable result. Even for $R=$ -3 a reasonable value for the center gain $g_{o}$ is found for low values ( 4 or 6 ) of $l_{\max }$. 
TABLE I

\begin{tabular}{r|c|c|c|c|c|}
\hline $\begin{array}{c}g_{0} \\
\text { in } \mathrm{cm}^{-1}\end{array}$ & $R=-3$ & -2 & -1 & 0 & 1 \\
\hline eff index & 260.54 & 182.21 & 256.49 & 131.99 & 118.14 \\
\hline $\begin{array}{r}\text { plane vave } \\
\ell_{\max }=10\end{array}$ & 206.55 & 182.22 & 156.50 & 132.01 & 118.18 \\
3 & 206.55 & 282.22 & 156.50 & 132.01 & 115.18 \\
6 & 206.55 & 182.22 & 156.50 & 132.01 & 118.18 \\
4 & 206.55 & 182.22 & 156.51 & 132.02 & 118.18 \\
2 & 206.69 & 182.33 & 156.58 & 132.05 & 118.20 \\
0 & 212.13 & 186.38 & 159.25 & 133.52 & 119.04
\end{tabular}

\begin{tabular}{r|r|c|c|c|c|}
\multicolumn{1}{c|}{$\begin{array}{c}w_{0} \\
\text { in } \mathrm{um}\end{array}$} & $R=-3$ & -2 & -1 & 0 & 1 \\
\hline eff index & 10.309 & 9.6861 & 6.7423 & 7.3184 & 6.0361 \\
\hline plane wave & & & & & \\
$i_{\max }=10$ & 10.381 & 9.7288 & 8.7606 & 7.3220 & 6.0353 \\
8 & 10.480 & 9.7505 & 8.7620 & 7.3217 & 6.0352 \\
6 & 10.217 & 9.7096 & $\varepsilon .7677$ & 7.3250 & 6.0368 \\
4 & 10.201 & 9.5191 & 8.6664 & 7.2944 & 6.0208 \\
2 & 17.711 & 12.6050 & 9.4898 & 7.5235 & 6.1516 \\
0 & 8.946 & 8.4135 & 7.6034 & 6.3744 & 5.2627 \\
\hline
\end{tabular}

Center gain go and Eull width at half intensity calculated for $\lambda=0.9 \mu \mathrm{m}, \mathrm{G}_{\text {reg }}=50 \mathrm{~cm}^{-1}, \alpha=20 \mathrm{~cm}^{-1}, \mathrm{n}_{1}=3.6, \mathrm{n}_{2}=3.4$, $a=0.2: \mathrm{m}, y_{0}=6$ in.

Inspection of the expansion coefficients shows a behavior similar to the results in Table $I$ of [7], i.e., an increase of $l_{\max }$ only gives very small changes of the coefficients $A_{l}$. As seen in Fig. 4(c) the consequences of higher values of $l_{\max }$ are a better convergence for low $y$-values and a shift of the peak to larger $y$-values.

From the calculations it is found that the boundary between convergence [as in Fig. 4(a)] and development of a peak [as in Fig. $4(\mathrm{c})$ ] can be described by the following empirical relation:

$$
\Gamma C \approx 0.2 \text {. }
$$

Below the curve given by (64) the planewave method may still be used to calculate center gain and intensity width, but it cannot give correct intensity distributions far from the center. In extreme cases of large negative $R$ values and small values for the thickness $d$, reliable results cannot be obtained even for $l_{\max }=10$. The validity of the expansion in (57) has been checked and it was found that the influence of a finite number of terms (i.e., $m=5$ ) was negligible except in a few extreme cases where the results were wrong anyway.

The bad agreement between the two methods reported in [7] is probably mainly due to the use of a formula for the gain $g_{o}$ somewhat different from (42).

As noted in [19] the far field in the $x=0$ plane is easily computed. We have considered the three examples from [19] which can be described by $\Delta n=0.1, d=0.1 \mu \mathrm{m}, R=-0.35$, -0.70 , and -1.05 . As these values lie below the $\Gamma C=0.2$ curve shown in Fig. 5, it is not surprising that the same convergence problems as seen for the near field occurs. The shape
$R=-1$

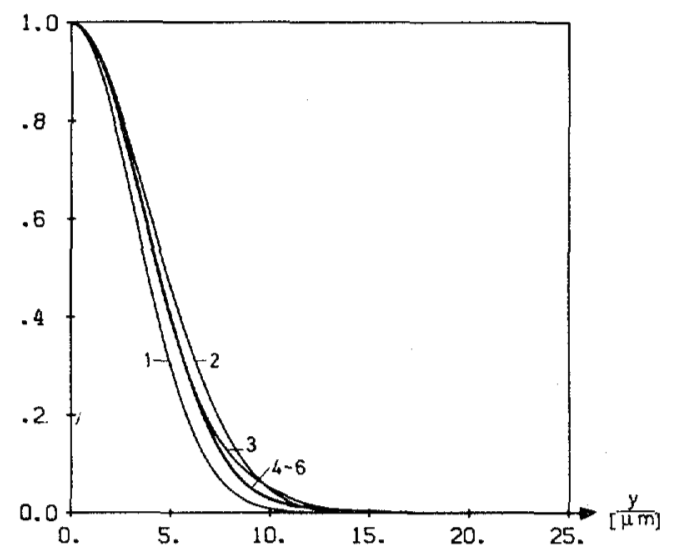

(a)

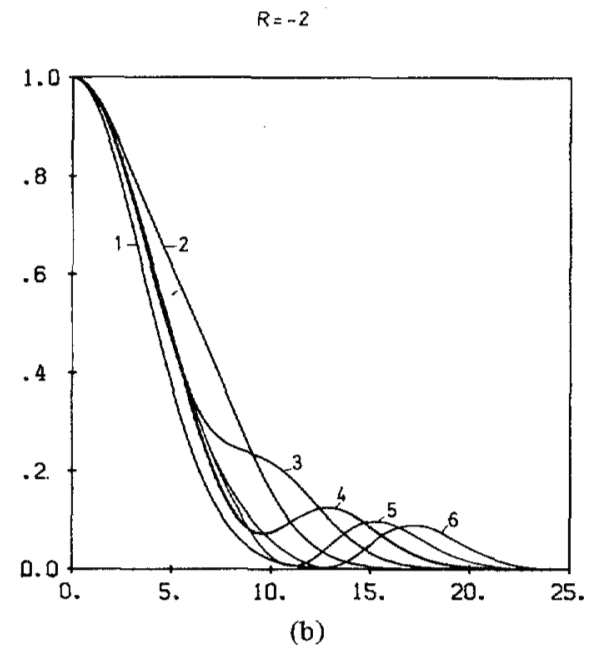

$R=-3$

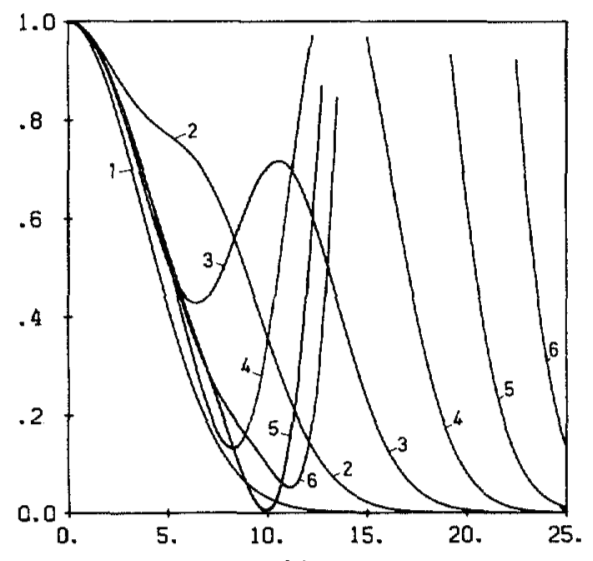

(c)

Fig. 4. Intensity distributions for three of the cases considered in Table I: (a) $R=-1$, (b) $R=-2$, and (c) $R=-3$. In all three cases the intensities are calculated for $l_{\max }=0,2,4,6,8$, and 10 .

of the far fields depends on the number of expansion terms in (52), and the shoulders reported in [19] are numerical. For an infinite parabolic profile in the active layer the effective index method always gives Gaussian far fields. For other profiles such as $\left(\cosh ^{2}\right)^{-1}[20],[21]$, Lorentzian [21], and truncated parabolic [21], more peculiar far fields appear. 


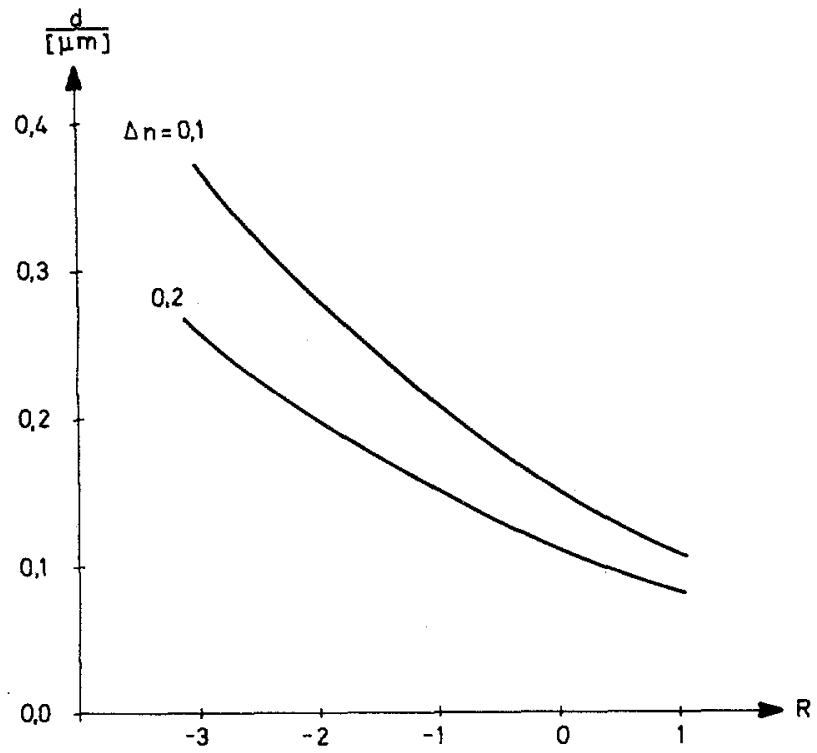

Fig. 5. The relation $\Gamma C=0.2$, where $\Gamma$ is defined in (10) and $C$ in (40), plotted as function of the parameter $R$ for $n_{1}=3.6$ and two values of $\Delta n=n_{1}-n_{2}$.

\section{Conclusion}

Application of the effective index method to the special case of a laser structure with a parabolic profile in the active layer results in simple formulas for center gain and half intensity width. These are compared with results obtained by a numerical planewave expansion method, and very good agreement is found for all cases where the numerical method gives reliable results. This agreement does not mean that the parabolic profile is a good description of the guiding in the laser, only that for this particular case the effective index method is both efficient and precise. The planewave expansion is only of practical interest for profiles where the field in the active layer and its Fourier transform can be expressed analytically. The good agreement for the parabolic case, however, gives good evidence for the validity of the effective index method also for other profiles.

The small difference in the expression used for the required gain in case of a broad active region ( $39 \mathrm{a}$ ) and the usual expression (39b) is only of theoretical interest and will not affect practical laser design.

\section{ACKNOWLEDGMENT}

The author would like to thank Dr. M. J. Adams, University of Southampton, Dr. G.H.B. Thompson, Standard Telecommunication Laboratories, and Dr. W. Streifer, Xerox Palo Alto Research Center for a large number of stimulating discussions on the effective index method. Furthermore, Dr. M. J. Adams, Dr. G. Jacobsen, Technical University of Denmark, and Dr. W. Nakwaski, Technical University of Łodz, Poland, are gratefully acknowledged for comments on the manuscript.

F. Birkedahl and J. Thode, both students at Electromagnetics Institute, Technical University of Denmark, are acknowledged for programming work.

\section{REFERENCES}

[1] R. M. Knox and P. P. Toulios, "Integrated circuits for the millimeter through optical frequency range," in Proc. MRI Symp. on Submillimeter Waves, J. Fox, Ed. Brooklyn, NY: Polytechnic Press, 1970.

[2] P. A. Kirkby and G.H.B. Thompson, "Channeled substrate buried heterostructure GaAs-(GaAl)As injection lasers," J. Appl. Phys., vol. 47 , pp. 4578-4589, 1976.

[3] T. L. Paoli, "Waveguiding in a stripe-geometry junction laser," IEEE J. Quantum Electron., vol. QE-13, pp. 662-668, 1977.

[4] P. M. Asbeck, D. A. Cammack, J. J. Daniele, and V. Kelbanoff, "Lateral mode behavior in narrow stripe lasers," IEEE J. Quantum Electron., vol. QE-15, pp. 727-733, 1979.

[5] J. Buus, "A model for the static properties of DH lasers," IEEE J. Quantum Electron., vol. QE-15, pp. 734-739, 1979.

[6] W. Streifer, R. D. Burnham, and D. R. Scifres, "Analysis of diode lasers with lateral spatial variations in thickness," Appl. Phys. Lett., vol. 37, pp. 121-123, 1980.

[7] J. K. Butler and J. B. Delaney, "A rigorous boundary value solution for the lateral modes of stripe geometry injection lasers," IEEE J. Quantum Electron., vol. QE-14, pp. 507-513, 1978.

[8] W. O. Schlosser, "Gain induced modes in planar structures," Bell Syst. Tech. J., vol. 52, pp. 887-908, 1973.

[9] M. J. Adams, An Introduction to Optical Waveguides. New York: Wiley, 1981, p. 159.

[10] W. Streifer and E. Kapon, "Application of the equivalent-index method to DH diode lasers," Appl. Opt., vol. 18, pp. 3724$3725,1979$.

[11] M. J. Adams, An Introduction to Optical Waveguides. New York: Wiley, pp. 208-209, 1981.

[12] J. Buus, "Detailed field model for DH stripe lasers," Opt. Quantum Electron., vol. 10, pp. 459-474, 1978.

[13] The fact that the difference between [5], [12] and other references, e.g., [3], [4], [6], [10] is due to the omission of the last term in (16) was pointed out by G.H.B. Thompson.

[14] G. B. Hocker and W. K. Burns, "Mode dispersion in diffused channel waveguides by the effective index method," Appl. Opt., vol. 16, pp. 113-118, 1977.

[15] E.A.J. Marcatili, "Dielectric rectangular waveguide and directional coupler for integrated optics," Bell Syst. Tech. J., vol. 48, pp. 2071-2102, 1969.

[16] K. Petermann, "Calculated spontaneous emission factor for double-heterostructure injection lasers with gain-induced waveguiding," IEEE J. Quantum Electron., vol. QE-15, pp. 566$570,1979$.

[17] M. Abramowitz and I. A. Stegun, Handbook of Mathematical Functions. New York: Dover, 1972.

[18] I. S. Gradshteyn and I. W. Ryzhik, Table of Integrals Series and Products. New York: Academic, 1965.

[19] J. K. Butler and J. B. Delaney, "Field solutions for the lateral modes of stripe geometry injection lasers," IEEE $J$, Quantum Electron., vol. QE-16, pp. 1326-1328, 1980.

[20] P. M. Asbeck, D. A. Cammack, and J. J. Daniele, "Non-gaussian fundamental mode patterns in narrow-stripe-geometry lasers," Appl. Phys. Lett., vol. 33, pp. 504-506, 1978.

[21] K. Petermann, "Some relations for the far-field distribution of semiconductor lasers with gain guiding," Opt. Quantum Electron., vol. 13, pp. 323-333, 1981.

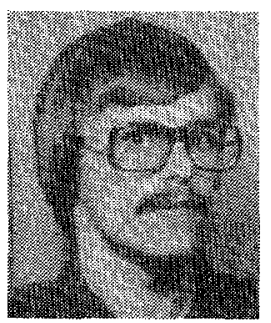

Jens Buus (S'76-M'79) was born in Copenhagen, Denmark, on August 8, 1952. He received the M.Sc. and Ph.D. degrees from the Technical University of Denmark, Lyngby, while doing research on the modeling of the properties of semiconductor lasers.

From March to June of 1979 he was a Research Associate at the University of Wales, Cardiff, Wales. Since August of 1979 he has been a Post Doctoral Research Associate at the Electromagnetics Institute, Technical University of Denmark, working on semiconductor lasers, in particular, waveguiding problems. 